\title{
Enhanced AC Quasi-steady State Cascading Failure Model for Grid Vulnerability Analysis under Wind Uncertainty
}

\author{
Mir Hadi Athari \\ Electrical and Computer Engineering \\ Virginia Commonwealth University \\ Richmond VA, USA \\ atharih@vcu.edu
}

\author{
Zhifang Wang \\ Electrical and Computer Engineering \\ Virginia Commonwealth University \\ Richmond VA, USA \\ zfwang@vcu.edu
}

\begin{abstract}
This paper presents several enhancements on a mixed OPF-stochastic cascading failure model to study the impacts of renewable energy resource uncertainty on grid vulnerability. The improved quasi-steady state (QSS) cascading failure model incorporates AC power flow calculations thus allowing us to simulate voltagerelated failures in the grid. The under-voltage load shedding (UVLS) relays are modeled along with a stochastic time-inverse overload relay to accurately simulate the protective system response. In addition, more realistic assumptions are considered in the modeling of wind power penetration using geographical information of grid topology and wind potential map for a given geographical area. The effectiveness of the proposed framework is evaluated on a 500-bus synthetic network developed based on the footprints of South Carolina. The enhanced model allows us to more accurately simulate cascades in the power system with high penetration of erratic renewables and identify weak points.
\end{abstract}

Keywords: Grid vulnerability, GIS information, QSS cascading failure model, Spatial correlation, Wind power

\section{Introduction}

Cascading failures in power grid is defined by the North American Electric Reliability Corporation (NERC) as: "The uncontrolled successive loss of Bulk Electric System Facilities triggered by an incident (or condition) at any location resulting in the interruption of electric service that cannot be restrained from spreading beyond a predetermined area" [1]. The electrical power system as a critical infrastructure plays a vital role in the economics, social life, and national security. Despite all preventive and protective measures taken by system operators, large-scale cascading failures leading to blackouts happen in power systems, though very rarely. However, the huge economic and social impacts of such events necessitate a systematic risk assessment for existing grid and the minimization of cascading risk is, therefore, a continuing area of research in both academic and industrial settings.

The research on cascading failure in the literature mainly focuses on modeling and analysis methods to accurately simulate cascading failure to assess the blackout risk for a given network [2]. There exist two major approaches in simulating cascading failure including dynamic transient models [3]-[8] and quasisteady state (QSS) models [9]-[19], where each have advantages and disadvantages. In the dynamic models, the dynamic components, such as rotating machines, exciters, and governors are modeled using differential equations. Also, to accurately predict the behavior of the system all the protective components of the system along with their dynamic behavior must be modeled. These add to the computational burden of the simulation especially for large cases which prevents running multiple Monte Caro (MC) simulations to assess the risk of blackout for different planning scenarios. In addition, the numerical failure in solving differential equations and the many assumptions made in the dynamic models decrease the accuracy of the results generated. The models in [3], [4] and the COSMIC model in [5], [6] are examples of research-grade dynamic cascading failure models and [7], [8] are examples of existing commercial simulation tools that have introduced dynamic simulation to their cascading failure analysis. On the other hand, the QSS models are widely used in the literature to study the cascading failure and evaluate the risk of large-scale blackouts. These models rely on the steady-state assumption for the system where the flow re-dispatch of the network is calculated based on power flow analysis. These model differ from each other in terms of the assumptions they make to simulate the cascading failure and the Power Flow (PF) model used. The representation of the transmission system can be based on the full version of PF equations (i.e., ACPF), or on the linearized version (i.e., DCPF). Although the DCPF guarantees convergence for the power flow and allows the simulation of failures beyond any topology changes for the grid, it lacks the information for voltage 
profiles thus making it impossible to take into account voltage-related failures. The ORNL-PSerc-Alaska (OPA) models in [9], [10], the random chemistry model in [11], the Markov-transition model in [12], and more recently the mixed OPF-stochastic model in [13], [14] are examples of the cascading failure models that employ DCPF. Whereas the AC OPA model in [15], Manchester model in [16], TRELSS model in [17], importance sampling model in [18], and more recently the AC-OPF-f model in [19] are among the models employing full AC power flow in the simulation of cascading failures.

Almost all of the above-mentioned models study the cascading failure and blackout risk for the traditional power systems where there is either zero or very low penetration of erratic renewable generation resources. However, as the penetration of renewables to the modern power systems increases, there is a need for studies that evaluate the vulnerability of the transmission network to cascading failure under the shifting energy portfolio. For example, Henneaux et al. studied the impact of thermal effects on the risk of blackout for increased wind farms [20]. Scala et al. in [21] found that the presence of fluctuations due to erratic renewable sources and customer demands increases the instability within an isolated segment of a power grid. In [14] we investigated the uncertainty injected from highly variable renewable energy resources to the grid and proposed a mixed OPF-stochastic cascading failure model based on DCPF that employs a stochastic tripping mechanism taking into account the uncertainty injected from wind generators. However, in this study, it is assumed that wind farms are randomly integrated into the grid by replacing conventional generators to reach a specific penetration ratio and the spatial correlation of wind farms are neglected.

Since, the preliminary results in [14] suggest that higher penetration of wind generation may lead to more severe cascading failure outcomes in terms of total load shedding and total tripping events, in this paper we make several enhancements in our model to achieve a more realistic assumption/modeling of uncertainty sources from renewables. Another enhancement is incorporating $\mathrm{AC}$ power flow in our analysis model to use voltage profiles for implementation of undervoltage load shedding (UVLS) relays. These improvements help us achieve a more accurate evaluation of grid vulnerability to cascading failure with expected renewable energy growth in the energy portfolio.

The rest of the paper is organized as follows. In section 2, we discuss how GIS information is used to enhance wind installation assumptions in our methodology. Section 3 presents all enhancement made on our cascading failure analysis method by incorporating AC power flow. Section 4 examines the application of the proposed methodology in a 500-bus synthetic power grid network and section 5 concludes the paper.

\section{Modeling Wind Power Installation}

In [14] we presented the preliminary analysis on the impacts of high penetration of renewable energy sources on grid vulnerability to cascading overload failures. In this model, the simulation of cascading failure was carried out based on flow re-dispatch using DCPF approximation and tripping of overloaded lines to predict the most probable cascade path for a given contingency scenario. The spatial correlation of installed wind generators has been neglected and it was assumed that every new wind installation randomly replaces a conventional generator in the original setting of the network. In this paper, we propose a methodology for grid vulnerability assessment studies with two major enhancement in the previous model in simulation inputs and cascading failure analysis method.

Our previous analysis on grid vulnerability suggests that increased uncertainty level injected from renewable energy resources as well as electrical loads may have an adverse impact on blackout size resulting from cascading failure in power systems. Therefore, it is important to improve our model so that it allows for more realistic assumptions/modeling of uncertainty sources from renewables in our analyses by integrating the geographical information of network topology and wind potential capacity into our simulation model. According to National Renewable Energy Laboratory (NREL) 2014 report [22], renewable generation accounts for $50 \%$ of the U.S. new energy installation capacity. Among various types of renewable generation, wind energy ranks second after hydropower in terms of percentage of total generation. Considering strictly increasing rate of wind power penetration to the national grid, it is critical to systematically evaluate the impacts this shifting energy portfolio would have on grid vulnerability.

To address this need, one should take into account the land potential of wind capacity to simulate the probable generation expansion planning scenarios in the near future more accurately. In other words, wind potential capacity maps such as the ones published by NREL can give useful insights on the probable location and capacity of the future wind farms integrating to the existing grid (Fig. 1) [23]. The potential wind capacity map shows the land area with a gross capacity factor of $35 \%$ and higher, which may be suitable for wind energy development. AWS Truepower produced the wind resource data with a spatial resolution of 200-m, which 
was binned into 20-km grid cells. Map shading shows the amount of area with the potential to be developed within each $20-\mathrm{km}$ cell: the darker the color, the larger the potentially developable area within each cell. These maps exclude areas that are prohibited by law from development, such as wilderness areas and national parks, and other areas unlikely to be developed, such as urban areas and water bodies. Potential wind capacity maps are provided for a 2014 industry standard wind turbine installed on a $110-\mathrm{m}$ tower, which represents plausible current technology options.

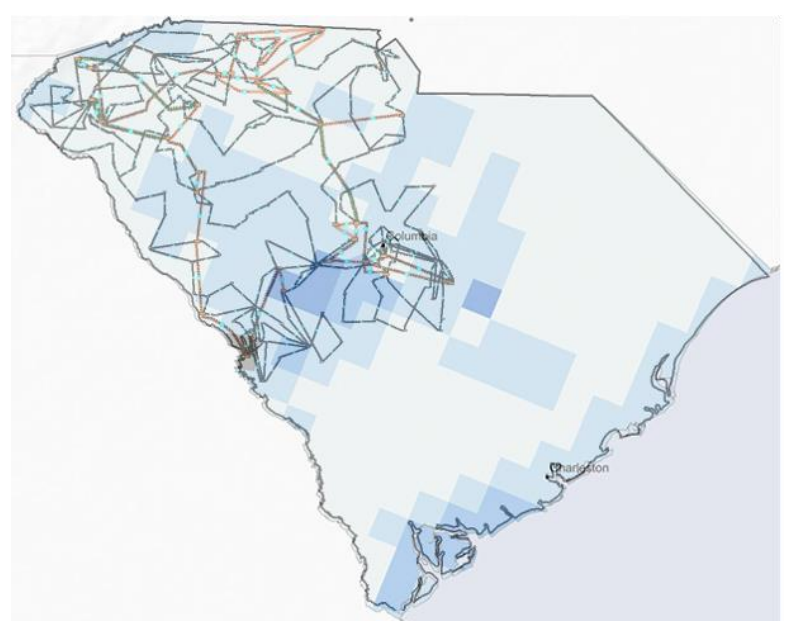

Figure 1. South Carolina 110-meter potential wind capacity map combined with ACTIVSg500 synthetic network with two voltage levels 138 and $230 \mathrm{kV}$ [23], [24].

Using the potential wind capacity map and geographical information of the grid topology, we can determine the possible point of interconnection for potential wind farms. For this, we calculate the direct distance of each potential area from all substations of the grid. Then we choose the closest substation within a predefined radius of the area, say $20 \mathrm{~km}$. Note that, this radius depends on various economic and technical parameters and is worthy of more investigation. Now, we have the potential area and substation pairs for the given network. Next, we sort the list of candidate substations based on their maximum potential wind power (MW) installation capacity (as shown in Table 1).

Inside every substation, there are multiple buses that can be selected as the point of coupling for wind farms. For this, we exclude load buses and select either generation or connection buses. Note that for the sake of consistency, we select buses with the same nominal voltage level throughout this process. The candidate buses for South Carolina 500-bus synthetic network have been highlighted in Table 1 .
Table 1. Potential substation and respective MW of wind for South Carolina 500 bus synthetic network

\begin{tabular}{|c|c|c|}
\hline Substation ID & Potential MW of Wind & Candidate Buses \\
\hline 4 & 695 & {$[\mathbf{8} ; 7 ; 9]$} \\
\hline 8 & 629 & {$[20 ; \mathbf{1 9}]$} \\
\hline 151 & 596 & {$[373 ; \mathbf{3 7 2} ; 371]$} \\
\hline 79 & 552 & {$[190 ; \mathbf{1 8 9}]$} \\
\hline 67 & 505 & {$[159 ; 158]$} \\
\hline 107 & 421 & {$[268 ; 267]$} \\
\hline 152 & 355 & {$[375 ; 374]$} \\
\hline 207 & 281 & 499 \\
\hline 44 & 252 & {$[105 ; 104]$} \\
\hline 45 & 210 & {$[107 ; 106]$} \\
\hline 80 & 163 & {$[192 ; 191]$} \\
\hline
\end{tabular}

The one-line diagram of ACTIVSg500 network which is a synthetic power system model that does not represent the actual grid is superimposed on the wind potential map for South Carolina in Fig. 1. The ACTIVSg500 is developed as part of the ARPA-E Grid Data research project and contains no Critical Energy/Electric Infrastructure Information (CEII) [24]. Fig. 2 shows the part of the network with a high concentration of wind farms. As an example to demonstrate our methodology we selected the top four locations to install wind farms. These locations are marked with green rectangles inside red ovals in Fig. 2.

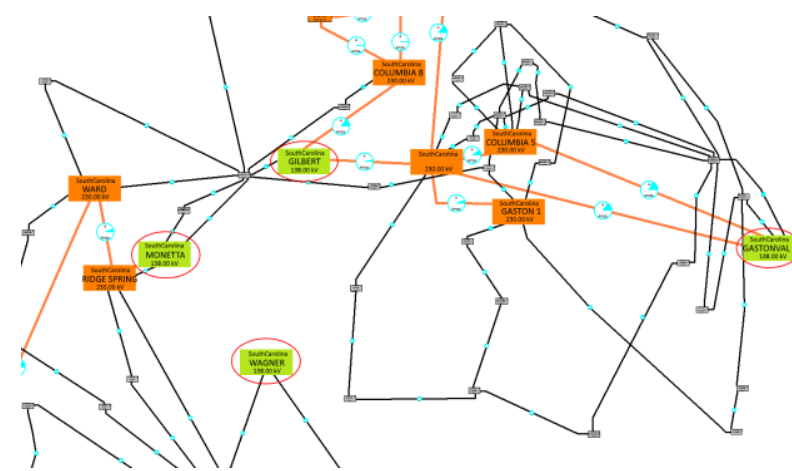

Figure 2. Location of four substations with integrated wind farms.

\section{Enhanced CF Analysis Model with Voltage Dynamics Using AC Power Flow}

Dynamic transient model and QSS model are the two most commonly used simulation approaches for 
cascading failure analysis in power systems. Each has its own pros and cons and focuses on different aspects of grid vulnerability analysis. The transient model includes the transient behavior of the system such as generator dynamics and possible dynamic simulation of load demand [16]. For this model, the mathematical formulation of the model is based upon a system of Differential Algebraic Equations (DAE) which comes from modeling the dynamics of machines, generator governor, and exciter systems. While this model allows for the detailed simulation of system transient behavior, it adds to the computational burden of cascading simulation, especially for the large-scale systems vulnerability analysis. In addition, numerical failures in solving the DAE system can greatly affect the simulation result and the size of the blackout, since for the network experiencing numerical failure a complete blackout is assumed. This illustrates a tradeoff that comes with using detailed nonlinear dynamic models: while the component models are more accurate, the many assumptions that are needed substantially impact the outcomes, potentially in ways that are not fully accurate. It is also found that the load models can substantially impact cascade sizes in transient dynamic models and different assumptions can lead to significant differences in the blackout risk assessment [6].

On the other hand, the QSS models are widely used to simulate cascading failures in power system and assess the vulnerability of the grid. These models rely solely on the steady state operation of the system after all transient dynamics of generator and loads have been settled. In these models, the flow re-dispatch is calculated based on PF analysis to determine the overloaded lines after every tripping event in the network. Usually, the DCPF is incorporated to decrease the computational expense and guaranteed convergence which comes at the price of approximated flat voltage profiles across the whole network.

Here we build upon our proposed CF model in [14] which is based on DCPF and stochastic line tripping method. We enhance our previous model by incorporating ACPF that allows for simulation of dynamic transition of system state variables such as voltage magnitude and angles and line flows. This, in turn, allows us to implement under-voltage and overvoltage load shedding relays to more accurately simulate the behavior of the protection system during the escalation phase of CF.

Fig. 3 shows the flowchart of the proposed CF model with ACPF and all enhancements applied over the DC model. The first enhancement comes from the integration of the geographical information for installed wind farms to account for spatial correlation of uncertainty injected from these highly variable sources. As discussed in section 2, this is accomplished by wind energy potential map and grid GIS information to effectively determine the point of interconnection for the wind farms and accurately model the injected uncertainty. All other enhancements come from the incorporation of full ACPF in the CF model where the flow re-dispatch is calculated by solving the full AC model. This provides us with the additional information necessary to model detailed protective scheme and corrective actions taken by system operators, thus allowing us to simulate voltage-related failures during a cascading failure. The key components of the proposed model include ACPF, stochastic time-inverse overload (STIO) relay, under-voltage load shedding (UVLS) relay, generation/load adjustment (power balance), island detection, and stochastic line trip mechanism. In this flowchart $U V L S_{\text {delay }}$ is the delay of UVLS relay in seconds, $L_{1}$ and $L_{2}$ are the first line index and the second line index for the $\mathrm{N}-2$ contingency, respectively, and $T_{0}$ is the initial contingency time. Trip $p_{l}^{\text {timer }}$ is line $l$ relay timer at each given time which is triggered when a line becomes overloaded. $Y(t)$ is the network admittance matrix at time $t, A$ is the adjacency matrix where $A_{i j}=$ 1 if nodes $i$ and $j$ are connected, 0 otherwise, $\Lambda^{-1}($. denotes the diagonal inverse matrix with a specific vector, and $z_{l}$ is the vector of branch impedances in the grid. $V_{\text {init }}(t)$ is the Newton-Raphson initial voltage guess at time $t . P_{g}(t)$ and $P_{L}(t)$ are matrices of generator output power and load demand power at time $t$. Next, the detailed modeling of each component is discussed.

\subsection{AC power flow}

The power flow problem is the computation of voltage magnitude and phase angle at each bus in a power system under balanced three-phase steady-state conditions. As a by-product of this calculation, real and reactive power flows in equipment such as transmission lines and transformers, as well as equipment losses, can be determined. For a power grid with $N$ nodes, the nodal equations for a power system network enforced by Kirchhoff's law are written as

$$
\boldsymbol{I}=\boldsymbol{Y}_{\text {bus }} \boldsymbol{V}
$$

where $I$ is the $N$ vector of source currents injected into each bus and $\boldsymbol{V}$ is the $N$ vector of bus voltages, and $\boldsymbol{Y}_{\text {bus }}$ is the network admittance matrix.

Then, the complex power delivered to bus $k$ can be written as: $S_{k}=P_{k}+j Q_{k}=V_{k} I_{k}^{*}$, with $I_{k}^{*}$ being the conjugate of the injected current at bus $k$. By taking the real and imaginary parts of the power balance equation and doing some simplifications, the nonlinear power flow equations are given by

$$
\begin{aligned}
& P_{k}=V_{k} \sum_{n=1}^{N} Y_{k n} V_{n} \cos \left(\delta_{k}-\delta_{n}-\theta_{k n}\right) \\
& =P_{G_{k}}-P_{D_{k}}
\end{aligned}
$$




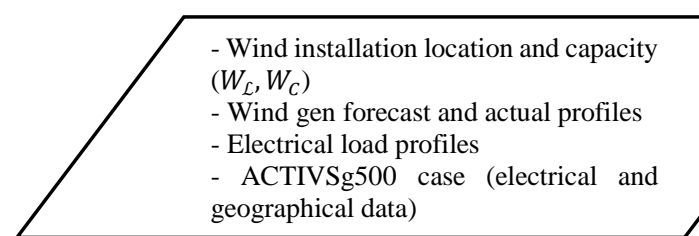

$\left(W_{\mathcal{L}}, W_{C}\right)$

- Wind gen forecast and actual profiles

- ACTIVSg500 case (electrical and geographical data)

Run OPF for the simulation period ( 2 hours) using

forecast profiles for loads and wind farms

- Save generation dispatch at each time step $\left(\operatorname{Gen}_{P}\right)$

- Save PV bus voltage settings $\left(V_{P V}\right)$

- Calculate and save line flow bandwidth $\left(B W_{l}\right)$

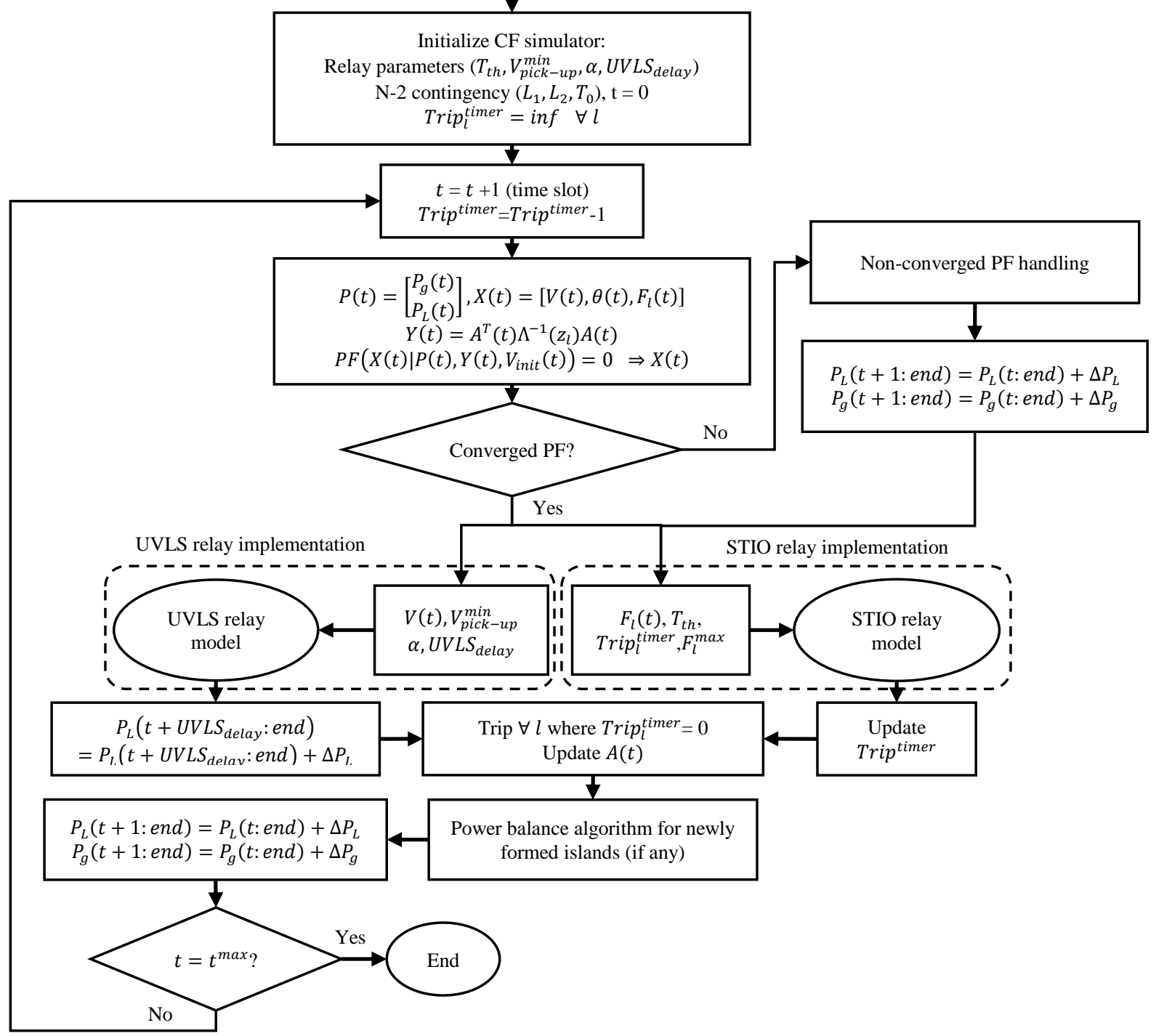

Figure 3. Flowchart of the proposed CF model with ACPF.

$$
\begin{aligned}
& Q_{k}=V_{k} \sum_{n=1}^{N} Y_{k n} V_{n} \sin \left(\delta_{k}-\delta_{n}-\theta_{k n}\right) \\
& =Q_{G_{k}}-Q_{D_{k}}
\end{aligned}
$$

where $S_{G_{k}}=P_{G_{k}}+j Q_{G_{k}}$ is the generation and $S_{D_{k}}=$ $P_{D_{k}}+j Q_{D_{k}}$ is the load demand at bus $k$. These nonlinear power balance equations are solved using iterative methods such as the Newton-Raphson algorithm [25]. In our CF model, at each time step, the full AC power flow is solved to find the system state variables, as well as line flows. Next, based on the system status, the overload and under/over-voltage relays operate as modeled next.

\subsection{Stochastic time-inverse overload relay}

In our mixed OPF-stochastic CF model the flow process of each line is assumed to be Gaussian where the normalized overload distance is calculated as $a_{l}=$ 
$\frac{F_{l}^{\max }-\mu_{F_{l}}(t)}{\sigma_{F_{l}}(t)}$, where $F_{l}^{\text {max }}$ is line capacity, $\mu_{F_{l}}(t)$ is average flow at time $t$, and $\sigma_{F_{l}}(t)$ is the variance of the flow process. Then, with a Gaussian assumption for the distribution of $F_{l}(t)$, the overloading probability $\rho_{l}(t)=p\left\{\left|F_{l}(t)\right|>F_{l}^{\max }\right\}$ can be calculated using Qfunction as below:

$$
\rho_{l}(t) \cong Q\left(a_{l}\right)
$$

where $Q(x)=\int_{x}^{\infty} e^{-t^{2} / 2} /(\sqrt{2 \pi}) d t$.

Next, using the normalized overload distance $\left(a_{l}\right)$ and overloading probability $\left(\rho_{l}\right)$ for each line we can calculate the mean overload time for flow process $F_{l}(t)$ as:

$$
\bar{\tau}_{l}^{u}=\frac{2 \pi \rho_{l} e^{a_{l}{ }^{2} / 2}}{B W_{l}}
$$

where $B W_{l}$ is the equivalent bandwidth of the flow process for the $l$ th line and can be calculated using the spectral power density (SPD) of the flow process [12], [14], [26].

These equations constitute the stochastic part of the overload relay model which allows for consideration of uncertainties injected from renewable energy sources to the line flows. The time-inverse delay algorithm is implemented by a counter function which is triggered when the line becomes overloaded and the time to trip is inversely proportional to the overload value and is determined based on the thermal stability of the overhead transmission lines as:

$$
t_{t r}=T_{t h \cdot} \cdot \ln \left(\frac{F^{2}-F_{o p}^{2}}{F^{2}-F_{\text {max }}^{2}}\right)
$$

where $F$ is overloaded line flow (p.u.), $F_{o p}$ is initial operating flow (p.u.), $F_{\text {max }}$ is the line flow threshold, and $T_{t h}$ is the thermal time constant which is related to conductor type and environmental parameters such as wind speed and ambient temperature [19]. If the line flow remains beyond the threshold, this timer will continue to count down from $t_{t r}$. Note that $t_{t r}$ is being updated regularly to account for variation of the flow due to variable renewable generation and this update procedure is with memory meaning that it takes into account the elapsed time since the first overload instance to account for the accumulative heat generated in the line. Finally, when setting the counter to $t_{t r}$, this value is compared to $\bar{\tau}_{l}^{u}$, if it is larger than $\bar{\tau}_{l}^{u}$, the trip timer is set to zero, otherwise, the trip timer is set to the relay time to trip $\left(t_{t r}\right)$. This tripping mechanism enables us to model the stochastic process of CF and identify the most probable path for its propagation.

\subsection{Under/over-voltage relay}

The AC power flow provides the network voltage profiles thus allowing us to employ UVLS relays. The
UVLS relay is incorporated in our CF modeling to account for the voltage control mechanism in the power system. This relay will shed a predefined percentage of the initial load $P_{D_{i}}$ at bus $i$ to avoid the onset of voltage instability and reduce system stress.

$$
\dot{P}_{D_{i}}=\alpha . P_{D_{i}} \text { if }\left|V_{i}\right| \leq c . V_{\text {pick-up }}^{\min }
$$

where $\alpha$ is the load shedding percentage (e.g. 25\%), $V_{\text {pick-up }}^{\min }$ is the minimum acceptable voltage magnitude of the bus $i$, and $c$ is a coefficient to determine the activation threshold for the UVLS relay. For the case study in this paper we set $\alpha=0.25, V_{\text {pick-up }}^{\text {min }}=$ 0.95 p.u., and $c=0.91$ according to [6].

The over-voltage relay is also incorporated and similar to UVLS relay it is activated when the voltage at bus $i$ rises above a threshold. These relays protect most generators while UVLS relays are mainly designed to protect large-capacity load motors [18]. For the overvoltage relay, the bus gets isolated instead and all the loads and generation at the bus are set to zero. Both over-voltage and under-voltage relays use a fixed time delay of $0.5 \mathrm{~s}$ to make sure that all transient dynamics of the voltage have been died out.

\subsection{Power balance and load shedding for islands}

Successive line tripping during the escalation phase of CF usually causes the formation of several islands in the power network. Usually, the generation and load of the formed islands become unbalanced which triggers the under-frequency load shedding (UFLS) relays to operate. In addition, the generator's governor tries to increase the generator output to compensate for the frequency drop in the system. All of these are considered in our CF model within the power balance algorithm for newly formed islands and the mother island proposed in [14]. After every tripping event, an island detection algorithm identifies new islands, if any, as well as isolated buses. Next, the power balance algorithm tries to maintain the balance between the generation and load by means of shedding actions or ramping up the generators.

\subsection{Non-convergent AC power flow}

Even after enforcing power balance for the islands formed in the network, the AC power flow may not converge. This is one of the challenges of incorporating the AC power flow calculation in $\mathrm{CF}$ simulation and could be due to various reasons. The most probable reason for non-convergent $\mathrm{AC}$ power flow is the case where the system load exceeds the steady-state loading limit. The steady-state loading limit is determined from 
a nose curve where the nose represents the maximum power transfer that the system can handle given a power transfer schedule. To determine the steady-state loading limit, the basic power flow equations

$$
g(x)=\left[\begin{array}{l}
P(x)-P^{i n j} \\
Q(x)-Q^{i n j}
\end{array}\right]=0
$$

are restructured with a scaling factor $\lambda$ as:

$$
f(x, \lambda)=g(x)-\lambda b=0
$$

where $x \equiv\left(\Theta, V_{m}\right)$, the vector of system state variables (i.e. voltage phase angles and magnitude), and $b$ is a vector of power transfer given by

$$
b=\left[\begin{array}{l}
P_{\text {target }}^{\text {inj }}-P_{\text {base }}^{\text {inj }} \\
Q_{\text {target }}^{\text {inj }}-Q_{\text {base }}^{\text {inj }}
\end{array}\right]
$$

where $P_{\text {base }}^{i n j}$ and $Q_{\text {base }}^{i n j}$ are injected real and reactive power for the base case, respectively (usually set to zero), and $P_{\text {target }}^{\text {inj }}$ and $Q_{\text {target }}^{\text {inj }}$ are target injected real and reactive power, respectively that for our case is the current dispatched power for the non-converged PF. The effects of the variation of loading or generation can be investigated using the continuation power flow (CPF) by composing the $b$ vector appropriately [28]. To check if this is the case, we run a CPF that gradually increase the loading/generation. If the resulting scaling factor $\left(\lambda_{\text {max }}^{c p f}\right)$ associated with the maximum loading that the system can handle is less than 1 , it indicates that the load for the case exceeds the steady-state loading limit, and loads must be scaled down at least by a factor of $\lambda_{\max }^{c p f}$ to get a convergent power flow solution.

\section{Case Study}

In this section, we evaluate the new proposed framework for grid vulnerability studies on ACTIVSg500 synthetic power grid with 597 branches [24]. The ACTIVSg500 is chosen because it offers detailed information on grid data including transmission line rates and geographical information of the network substations which is based on the footprints of South Carolina. The power systems are required by NERC standards to be $\mathrm{N}-1$ secure meaning that the system should be able to recover from any disturbance resulting from outage of an element in the system. Therefore, in order to trigger a cascade of events, at least two elements need to be taken out. To examine the proposed $\mathrm{CF}$ model with all discussed enhancements, three $\mathrm{N}-2$ contingency scenarios are considered to study the overall grid vulnerability and blackout size. These scenarios are selected in a way that the operation of different relays considered in the model (UVLS and STIO) can be observed. Note that for overall grid vulnerability analysis, it is necessary to run multiple Monte Carlo simulations and derive the distribution of the blackout size which is the subject of our future work. For all scenarios, four wind farms are installed in the buses identified in section 2. Table 2 shows the maximum wind capacity (MW), installation factor, and rated power of installed wind farms.

The three $\mathrm{N}-2$ contingency scenarios include:

Scenario 1: lines 95 and 231 are tripped at $t=4 \mathrm{~min}$.

Scenario 2: lines 63 and 231 are tripped at $t=4 \mathrm{~min}$.

Scenario 3: lines 193 and 234 are tripped at $t=4 \mathrm{~min}$.

Note that in the new time-delayed overload relay implementation, tripping multiple lines at one instance is possible because of the memory operation. Therefore, the state of the system and probability of a line to get tripped depend both on the current state of the system as well as its past states. In other words, our CF model is not a Markovian process anymore. Table 3 shows the statistics of the three $\mathrm{N}-2$ contingency scenarios. As expected, the more line outage happens in the network the more islands are formed and consequently the more load shedding becomes necessary to maintain the power balance of each island. This is not necessarily true for UVLS though. For the second scenario, we have a total of $173 \mathrm{MW}$ UVLS which is larger than both other scenarios. This is due to the independent operation of under-voltage relays which are triggered by a certain threshold for every voltage profile.

Table 2. Characteristics of Installed Wind Farms at ACTIVSg500 Synthetic Network

\begin{tabular}{|c|c|c|c|}
\hline $\begin{array}{c}\text { Bus } \\
\text { number }\end{array}$ & $\begin{array}{c}\text { Max wind } \\
\text { potential } \\
\text { (MW) }\end{array}$ & $\begin{array}{c}\text { Installation } \\
\text { factor }\end{array}$ & $\begin{array}{c}\text { Rated power } \\
\text { of wind farm } \\
\text { (MW) }\end{array}$ \\
\hline 8 & 695 & 0.15 & 104.25 \\
\hline 19 & 629 & 0.15 & 94.35 \\
\hline 372 & 596 & 0.18 & 107.28 \\
\hline 189 & 552 & 0.20 & 110.40 \\
\hline
\end{tabular}

Table 3. Results of Three N-2 Contingency Scenarios

\begin{tabular}{|c|c|c|c|c|}
\hline N-2 Contingency & $\begin{array}{c}\text { Total } \\
\text { trip } \\
\text { count }\end{array}$ & $\begin{array}{c}\# \text { of } \\
\text { formed } \\
\text { islands }\end{array}$ & $\begin{array}{c}\text { Total } \\
\text { LS } \\
(\%)\end{array}$ & $\begin{array}{c}\text { Total } \\
\text { UVLS } \\
\text { (MW) }\end{array}$ \\
\hline Sncenario1: $\{95,231\}$ & 62 & 16 & 48.7 & 326 \\
\hline Sncenario $2:\{63,231\}$ & 51 & 12 & 44.6 & 348 \\
\hline Sncenario3: $\{193,234\}$ & 41 & 7 & 38.2 & 38 \\
\hline
\end{tabular}

Fig. 4 shows the evolution process of the three scenarios and the total load shedding for them. All three curves are comparable with typical cascade evolution curves recorded in history in terms of rate of the outage. They usually consist of a slow start, the escalation 
phase, and settlement. However, sometimes after one or two line outage, the escalation phase starts (scenario 1). The impact of time-delay model for overload relay is visible in the evolution curve of the second scenario where at $t=20 \mathrm{~min}$ we see a pause in trips but when the timers for multiple overloaded lines reach zero, the second escalation phase starts $(t=36 \mathrm{~min})$.

As extensively discussed in [29], currently there is no standard procedure for benchmarking and validating cascading failure models. Since no historical data for the chosen test system is available, the direct comparison and validation with realistic settings is not possible. However, a recommended procedure is to compare statistics of the cascading failure simulation results such as expected demand loss, distribution of demand loss, distribution of lines outaged and critical lines with those of other methods. Due to different assumptions in various cascading failure models, even these statistics may suggest big differences in the risk of blackouts calculated by each model. Therefore, here we only compare the overall metrics of the cascading failure results with historical events. For further analysis and model validation, a large number of Monte Carlo simulations under different operation conditions are necessary. In this paper we introduce our enhanced QSS model and the validation and benchmarking of the model is the subject of our ongoing efforts.

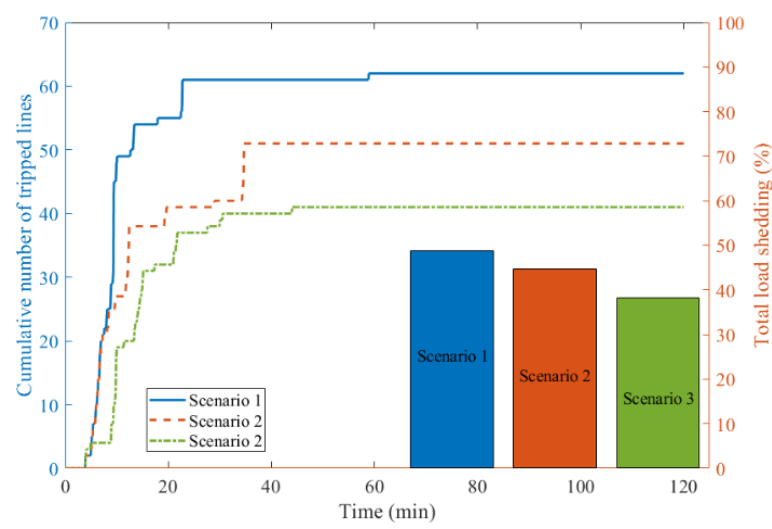

Figure 4. The evolution process of CFs for different scenarios.

Fig. 5 demonstrates the performance of the UVLS relay during $\mathrm{CF}$. At around minute 14 , due to a line trip in the network, the voltages on bus 418 and bus 341 start to drop. When the voltage drops below the UVLS relay activation threshold (0.87 p.u.) the relay starts to shed the load on the two buses by $25 \%$ for each time step to recover the voltage. After about 30 seconds, the load on bus 418 drops to $4.8 \mathrm{MW}$ which helps boost the voltage to 0.88 p.u. Then, the UVLS stops load shedding. However, for bus 341, the load shedding continues until all the load on the bus is shut down before boosting the voltage above the threshold.

This is an example of a condition during CF where voltage related failures lead to further load shedding in order to maintain voltage stability of the network and prevent a voltage collapse. Obviously, this condition could not be simulated based on the DC model and this confirms that the DCCF models may underestimate the severity of blackouts for real scenarios.
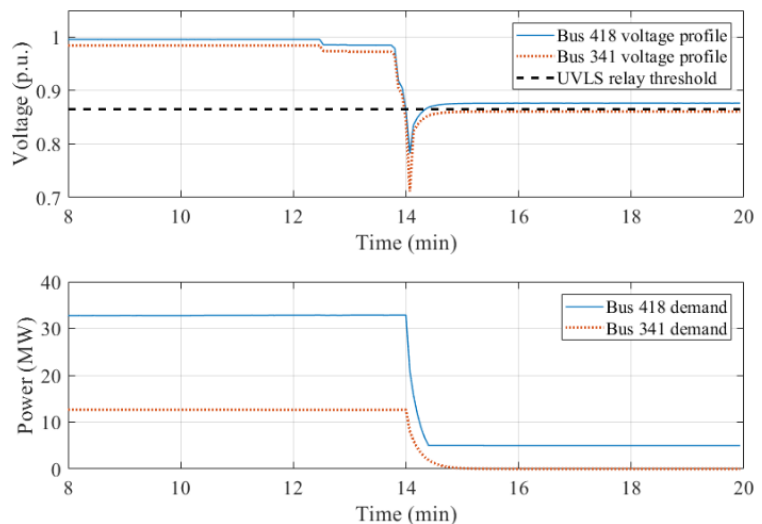

Figure 5. Voltage and load profiles for two select buses during failures.

\section{Conclusions and future work}

In this paper, we proposed an enhanced version of our methodology to study the grid vulnerability to cascading failure under high penetration of renewable energy sources. We improve our methodology by introducing more accurate evaluation/assumption of wind energy penetration to the grid using the geographical information of grid topology and wind potential capacity maps for a certain geographic area. We also improve our quasi-steady state (QSS) cascading failure model by incorporating the full AC power flow solution which provides the voltage profiles for implementation of under-voltage load shedding (UVLS) relays. One of the most common reasons for nonconvergent power flow problems is identified as the grid steady state loading limit violation and addressed by load shedding. The uncertainty injected from renewable energy is taken into consideration by stochastic timeinverse relay where the line tripping is performed based on overloading probability and thermal stability of the overhead transmission lines. Three N-2 contingency scenarios are simulated to examine the performance of the proposed methodology.

The validation and benchmarking of the model and sensitivity analysis of cascading outage impact based on varying wind penetration level are the subjects of our future work. 


\section{Acknowledgment}

The work in this study was sponsored in part by DoE through the ARPA-E program under grant number DEAR0000714.

\section{References}

[1] R. Fitzmaurice, E. Cotilla-Sanchez, and P. Hines, "Evaluating the impact of modeling assumptions for cascading failure simulation," in 2012 IEEE Power and Energy Society General Meeting, 2012, pp. 1-8.

[2] Vaiman et al., "Risk Assessment of Cascading Outages: Methodologies and Challenges," IEEE Trans. Power Syst., vol. 27, no. 2, pp. 631-641, May 2012.

[3] H. T. Ma, M. L. Crow, B. H. Chowdhury, and A. Lininger, "Cascading Line Outage Prevention with Multiple UPFCs," in 2007 39th North American Power Symposium, 2007, pp. 273-278.

[4] D. Fabozzi and T. Van Cutsem, "Simplified time-domain simulation of detailed long-term dynamic models," in 2009 IEEE Power \& Energy Society General Meeting, 2009, pp. 1-8.

[5] C. Parmer, E. Cotilla-Sanchez, H. K. Thornquist, and P. D. H. Hines, "Developing a dynamic model of cascading failure for high performance computing using trilinos," in Proceedings of the first international workshop on High performance computing, networking and analytics for the power grid - HiPCNA-PG '11, 2011, p. 25.

[6] J. Song, E. Cotilla-Sanchez, G. Ghanavati, and P. D. H. Hines, "Dynamic Modeling of Cascading Failure in Power Systems," IEEE Trans. Power Syst., vol. 31, no. 3, pp. 2085-2095, May 2016.

[7] J. P. Antoine and M. Stubbe, "EUROSTAG, software for the simulation of power system dynamics. Its application to the study of a voltage collapse scenario," in Interactive Graphic Power System Analysis Programs, IEE Colloquium on, 1992, pp. 1-5.

[8] N. Bhatt et al., "Assessing vulnerability to cascading outages," in 2009 IEEE/PES Power Systems Conference and Exposition, 2009, pp. 1-9.

[9] I. Dobson, B. A. Carreras, V. Lynch, and D. Newman, “An initial model for complex dynamics in electric power system blackouts," in Hawaii International Conference On System Sciences HICSS, 2001.

[10]Shengwei Mei, Fei He, Xuemin Zhang, Shengyu Wu, and Gang Wang, "An Improved OPA Model and Blackout Risk Assessment," IEEE Trans. Power Syst., vol. 24, no. 2, pp. 814-823, May 2009.

[11]M. J. Eppstein and P. D. H. Hines, "A 'Random Chemistry' Algorithm for Identifying Collections of Multiple Contingencies That Initiate Cascading Failure," IEEE Trans. Power Syst., vol. 27, no. 3, pp. 1698-1705, Aug. 2012.

[12]Z. Wang, A. Scaglione, and R. J. Thomas, "A MarkovTransition Model for Cascading Failures in Power Grids," in 2012 45th Hawaii International Conference on System Sciences, 2012, pp. 2115-2124.
[13]M. H. Athari and Z. Wang, "Studying Cascading Overload Failures under High Penetration of Wind Generation," 2017 IEEE Power Energy Soc. Gen. Meet. (PES GM).

[14]M. H. Athari and Z. Wang, "Impacts of Wind Power Uncertainty on Grid Vulnerability to Cascading Overload Failures," IEEE Trans. Sustain. Energy, vol. 9, no. 1, pp. 128-137, Jan. 2018.

[15]Shengwei Mei, Yixin Ni, Gang Wang, and Shengyu Wu, "A Study of Self-Organized Criticality of Power System Under Cascading Failures Based on AC-OPF With Voltage Stability Margin," IEEE Trans. Power Syst., vol. 23, no. 4, pp. 1719-1726, Nov. 2008.

[16]D. P. Nedic, I. Dobson, D. S. Kirschen, B. A. Carreras, and V. E. Lynch, "Criticality in a cascading failure blackout model," Int. J. Electr. Power Energy Syst., vol. 28, no. 9, pp. 627-633, Nov. 2006.

[17]M. P. Bhavaraju and N. E. Nour, "TRELSS: A computer program for transmission reliability evaluation of largescale systems," Electric Power Research Inst., Palo Alto, CA (United States); Public Service Electric and Gas Co., Newark, NJ (United States), 1992.

[18]Q. Chen and L. Mili, "Composite Power System Vulnerability Evaluation to Cascading Failures Using Importance Sampling and Antithetic Variates," IEEE Trans. Power Syst., vol. 28, no. 3, pp. 2321-2330, Aug. 2013

[19]W. Ju, K. Sun, and R. Yao, "Simulation of Cascading Outages Using a Power-Flow Model Considering Frequency," IEEE Access, vol. 6, pp. 37784-37795, 2018.

[20] P. Henneaux, P.-E. Labeau, and J.-C. Maun, "Blackout Probabilistic Risk Assessment and Thermal Effects: Impacts of Changes in Generation," IEEE Trans. Power Syst., vol. 28, no. 4, pp. 4722-4731, Nov. 2013.

[21]A. Scala, S. Pahwa, and C. Scoglio, "Cascade failures and distributed generation in power grids," Int. J. Crit. Infrastructures, vol. 11, no. 1, pp. 27-35, 2015.

[22]P. Beiter, "2014 Renewable Energy Data Book," 2015.

[23]"WINDExchange: Wind Energy in South Carolina." [Online]. Available: https://windexchange.energy.gov/states/sc. [Accessed: 25-Jan-2018].

[24]A. B. Birchfield, T. Xu, K. M. Gegner, K. S. Shetye, and T. J. Overbye, "Grid Structural Characteristics as Validation Criteria for Synthetic Networks," IEEE Trans. Power Syst., vol. 32, no. 4, pp. 3258-3265, Jul. 2017.

[25]A. J. Wood and B. F. Wollenberg, "Power system generation, operation and control," John Wiley, New York, 1984.

[26]M. H. Athari and Z. Wang, "Modeling the Uncertainties in Renewable Generation and Smart Grid Loads for the Study of the Grid Vulnerability," 2016 IEEE Power Energy Soc. Innov. Smart Grid Technol. Conf., 2016.

[27] R. Thrash, Overhead Conductor Manual 2nd Edition. Southwire Company, 2007.

[28]V. Ajjarapu and C. Christy, "The continuation power flow: a tool for steady state voltage stability analysis," IEEE Trans. Power Syst., vol. 7, no. 1, pp. 416-423, 1992.

[29]J. Bialek et al., "Benchmarking and Validation of Cascading Failure Analysis Tools," IEEE Trans. Power Syst., vol. 31, no. 6, pp. 4887-4900, Nov. 2016. 